Old Dominion University

ODU Digital Commons

2012

Plantar Vibrotactile Detection Deficits in Adults with Chronic Ankle Instability

Matthew C. Hoch

Patrick O. McKeon

Richard D. Andreatta

Follow this and additional works at: https://digitalcommons.odu.edu/hms_fac_pubs Part of the Exercise Science Commons, and the Sports Sciences Commons 


\title{
Plantar Vibrotactile Detection Deficits in Adults with Chronic Ankle Instability
}

\author{
MATTHEW C. HOCH ${ }^{1}$, PATRICK O. MCKEON ${ }^{2}$, and RICHARD D. ANDREATTA ${ }^{3}$ \\ ${ }^{1}$ Department of Human Movement Sciences, College of Education, Old Dominion University, Norfolk, VA; ${ }^{2}$ Division \\ of Athletic Training, College of Health Sciences, University of Kentucky, Lexington, KY; and ${ }^{3}$ Division of Communication \\ Sciences \& Disorders, College of Health Sciences, University of Kentucky, Lexington, KY
}

\begin{abstract}
HOCH, M. C., P. O. MCKEON, and R. D. ANDREATTA. Plantar Vibrotactile Detection Deficits in Adults with Chronic Ankle Instability. Med. Sci. Sports Exerc., Vol. 44, No. 4, pp. 666-672, 2012. Purpose: The purpose of this study was to investigate the vibrotactile detection thresholds of the plantar cutaneous afferents in subjects with chronic ankle instability compared with healthy control subjects. Methods: Eight adults with chronic ankle instability and eight adults with no ankle sprain history participated. Vibrotactile detection thresholds were assessed using a mechanical stimulus generator system, mounted onto an articulated microscope arm, which delivered sinusoidal vibrotactile inputs to the foot sole at three different sites: head of the first metatarsal, base of the fifth metatarsal, and the heel. Vibrotactile stimulation was delivered at a range of test frequencies that corresponded to the known responsiveness of cutaneous mechanoreceptors in the glabrous skin of the foot sole (10, 25, and $50 \mathrm{~Hz})$. Probe displacement measures (dB) from the last eight displacement trials that contained $50 \%$ positive detection responses were averaged to obtain a single threshold estimate for each test frequency and site combination. Results: The results of this study indicate that no significant group-by-site interactions were found for any test frequencies $(P>0.29)$. However, group main effects were present at the $10-\mathrm{Hz}(P<0.0001), 25-\mathrm{Hz}(P=0.03)$, and $50-$ $\mathrm{Hz}(P=0.04)$ test frequencies, indicating that subjects with chronic ankle instability had significantly higher detection thresholds or less sensitivity when stimulation sites were pooled. Conclusions: The results of this study indicate that subjects with chronic ankle instability may demonstrate decreased sensitivity on the plantar surface of the foot. These alterations in plantar cutaneous somatosensation may help explain the underlying mechanisms associated with the prolonged sensorimotor system impairments in postural control and gait commonly exhibited by people with chronic ankle instability. Key Words: ANKLE SPRAIN, SENSORIMOTOR SYSTEM, SOMATOSENSORY, LOWER EXTREMITY
\end{abstract}

$\Lambda$ nkles sprains are the most frequently occurring injury in the physically active (13). It is estimated that up to $70 \%$ of individuals who sustain a single ankle sprain experience residual symptoms, recurrent instability, and recurring sprains (1). This health condition, known as chronic ankle instability (CAI), is associated with decreased functional capacity (40) and has been linked to posttraumatic ankle osteoarthritis (35). Functional loss and repetitive injury are thought to occur from the combination of mechanical and functional deficits of the ankle joint and surrounding structures (12). Mechanical impairments include loss of range of motion, arthrokinematic alterations, ligamentous laxity, and degenerative changes (12). Functional

Address for correspondence: Matthew C. Hoch, Ph.D., ATC, Department of Human Movement Sciences, College of Education, Old Dominion University, Student Recreation Center, Room 1005, Norfolk, VA 23529; E-mail: mhoch@odu.edu.

Submitted for publication April 2011

Accepted for publication September 2011.

0195-9131/12/4404-0666/0

MEDICINE \& SCIENCE IN SPORTS \& EXERCISE E

Copyright (C) 2012 by the American College of Sports Medicine

DOI: 10.1249/MSS.0b013e3182390212 impairments are sensorimotor deficits that affect stability during functional movement including postural control, neuromuscular control, and proprioception (12). Although extensive research has investigated the functional impairments experienced by those with CAI, the underlying mechanism of these deficits remain poorly understood (12).

Somatosensory input from articular, musculotendinous, and cutaneous mechanoreceptors is important for maintaining gait and upright posture (23). In people with CAI, investigations into somatosensory alterations have focused primarily on changes arising from articular receptors located in the lateral ankle ligaments $(18,22)$. On the basis of inconsistent and conflicting results, other sources of afferent information need to be investigated. An important source of contextual somatosensory information is the plantar cutaneous receptors because they are the primary interface between the individual and the environment while standing and during locomotion (24). The areas of the plantar surface that seem to be the most important to explore are the heel, the base of the fifth metatarsal, and the head of the first metatarsal because these areas provide boundary relevant information such as plantar pressures during gait (27). Those with CAI have demonstrated altered plantar pressure distributions during gait (25) as well as lower time-to-boundary postural control associated with anterior displacement in the 
center of pressure $(19,28)$. On the basis of these findings, it could be speculated that alterations in the transmission of boundary relevant information from the plantar surface could be a contributing factor to several of the spatiotemporal deviations in gait and postural control associated with CAI.

Although the ankle has been purported to be the center for somatosensory alterations in people with CAI, reduced sensitivity from the plantar cutaneous receptors has been associated with deleterious alterations in postural control and gait after laboratory-induced anesthesia of the plantar surface, in normal aging, and in health conditions associated with peripheral neuropathy $(16,21)$. Despite these findings, few studies have specifically investigated changes in vibrotactile sensitivity after lower extremity injury. Individuals with a history of lower extremity musculoskeletal conditions have been reported to have reduced cutaneous vibrotactile detection thresholds at multiple sites on the lower extremity $(6,31,32)$; however, the plantar cutaneous receptors have not yet been explored. Exploring alterations in plantar cutaneous somatosensation is important for musculoskeletal research because this glabrous skin is primarily populated with slowly adapting $\mathrm{A} \beta$ low-threshold cutaneous receptors that transmit movement-related information (15). In addition, these receptors may be a source of essential noise which contributes to the overall complexity and functional variability of the sensorimotor system (7). Systematically examining plantar cutaneous vibrotactile detection thresholds may shed insight to the functional impairments and sensorimotor system constraint experienced by patients with CAI.

Vibrotactile detection threshold assessment has been demonstrated to be a reliable and well-controlled method of assessing the health and integrity of somatosensory pathways in humans (36). Assessing vibrotactile aspects of plantar surface acuity rather than traditional clinical measures of tactile sensation such as Semmes-Weinstein monofilaments or pressure algometry is an advantageous first step in this research because of the ability to purposefully manipulate and more precisely control the frequency, intensity, phase, and duration of the stimulus. The ability to adjust these parameters provide for a more sensitive means to identify subtle alterations in perceptual function. Evaluating plantar cutaneous somatosensory function in people with CAI may lend important insight as to how these individuals organize and use peripheral sources of cutaneous afferent information to maintain gait and posture, which could have implications for the evaluation and rehabilitation of this health condition. Therefore, the purpose of this study was to examine plantar cutaneous vibrotactile detection thresholds in subjects with CAI compared with healthy adults using a range of test frequencies that correspond to the known sensitivity of cutaneous mechanoreceptor populations (4).

\section{METHODS}

Participants. Eight adults with CAI (five males and three females; age $=26 \pm 3 \mathrm{yr}$, height $=176.11 \pm 3.47 \mathrm{~cm}$, weight $=88.26 \pm 15.64 \mathrm{~kg}$ ) and eight adults with no history of ankle sprain (five males and three females; age $=29 \pm$ $5 \mathrm{yr}$, height $=169.41 \pm 7.50 \mathrm{~cm}$, weight $=70.97 \pm 10.87 \mathrm{~kg}$ ) volunteered to participate. Inclusion criteria for the CAI group consisted of reporting a history of at least one ankle sprain, at least two episodes of "giving way" within the past 3 months, and answering "yes" to 4 or more questions on the Ankle Instability Instrument (8). In addition, subjects included in the CAI group reported disability scores of $\leq 90 \%$ on the Foot and Ankle Ability Measure (FAAM) and a score of $\leq 80 \%$ on the FAAM Sport (17). The Ankle Instability Instrument is a 12 -item questionnaire used to determine the presence of CAI based on the following factors: severity of initial ankle sprain, history of ankle instability, and instability during activities of daily life (8). The FAAM is designed to quantify activity limitations and participation restrictions in the previous week associated with health conditions affecting the foot and ankle. The activity of daily living portion of FAAM contains 21 activity-related items, whereas the FAAM Sport subscale contains 8 sportrelated items (17). In the event an individual reported bilateral CAI, the limb reporting the most disability on the FAAM instruments was included in the study. Subjects reported an average of $8 \pm 6$ total ankle sprains. The average number of self-reported episodes of giving way during the previous 3 months was $6 \pm 6$. The average FAAM score was $80.0 \% \pm 10.3 \%$, and the average FAAM Sport score was $62.5 \% \pm 15.4 \%$.

Inclusion criteria for control subjects included no history of ankle sprains and no reported disability on the FAAM and FAAM Sport (100\%). Exclusion criteria for both groups consisted of reporting an acute ankle sprain within the past $6 \mathrm{wk}$, a previous history of lower extremity surgeries, lower extremity injuries within the past 6 months (other than ankle sprains), diabetes, neuropathies, or other health conditions known to affect cutaneous sensation. Before participation, all subjects provided written informed consent, which was approved by the university's institutional review board.

Cutaneous stimulation. A mechanical stimulus generator system, mounted onto an articulated microscope arm (2), delivered sinusoidal vibrotactile inputs to the foot sole at three different sites: head of the first metatarsal, base of the fifth metatarsal, and the heel (Fig. 1). Vibrotactile inputs were delivered separately to each site in a randomized order. The stimulus system consisted of a Bruel and Kjaer Model 4810 Minishaker (Norcross, GA), a flat-surfaced nylon stimulus probe (surface area $=0.5 \mathrm{~cm}^{2}$ ), and a rigid surround (17-mm OD with a probe-surround gap $\approx 1 \mathrm{~mm})$. A Schaevitz (Fairfield, NJ) microminiature linear variable differential transformer (LVDT) was serially coupled to the outboard end of the Minishaker to provide displacement information of the probe with $1-\mu \mathrm{m}$ resolution. The output of an arbitrary digital waveform generator (Wavetek Model 29; Everett, WA) was conditioned by a power amplifier (Bruel and Kjaer Model 2706) and provided the input signal to the Minishaker. Synthesized waveforms from the 

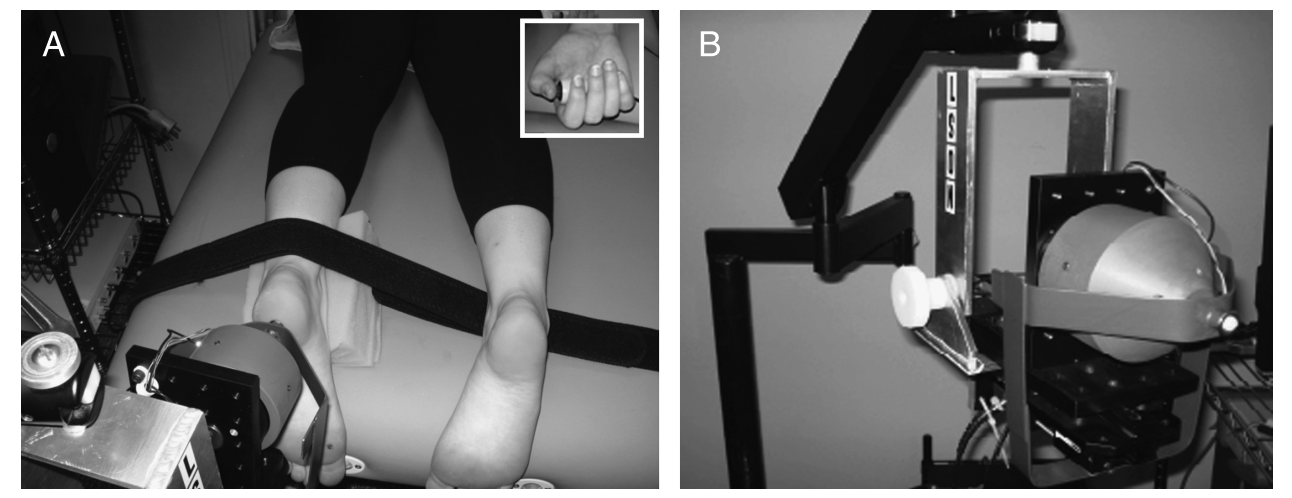

FIGURE 1-A, Positioning of the subject for assessing vibrotactile detection thresholds on the plantar surface using a custom-built mechanical stimulus generator system. B, Close-up view of the Minishaker with a $0.5-\mathrm{cm}^{2}$ probe and rigid surround. C, Close-up view of the handheld trigger used to register the subject's perceptual response to stimuli.

waveform generator were $1 \mathrm{~s}$ duration with a $150-\mathrm{ms}$ linear rise-fall decay to eliminate the possibility of on/off mechanical transients. Probe displacement signals from the LVDT were digitized at $5 \mathrm{kHz}$ on a 16-bit A/D (ADInstruments, Colorado Springs, CO). Probe displacement output was digitized in calibrated units of micrometers. General system calibration checks were performed by the investigators before subject testing to ensure consistency and accuracy in the output of the LVDT.

Vibratory detection threshold testing. Subjects were positioned in prone position with a prone pillow supporting the upper torso and head (Fig. 1). The limb included in the study was secured by a customized foam support with a series of straps over the triceps surae complex, foam support, and the toes to stabilize the foot and provide a consistently taut plantar surface to allow for orthogonal probe placement at each stimulation site. Subjects were instructed to remain relaxed and limit movement as much as possible during the psychophysical testing periods. The rigid surround about the contactor probe was then placed on a given stimulation site with a $1000-\mu \mathrm{m}$ contactor preload indentation. The same preload indentation was used at all stimulation sites and all input frequencies to ensure consistency in the magnitude of contact between the apparatus and foot sole surface.

Once subjects were secured in the testing position, vibrotactile stimulation was delivered to each of the three stimulation sites. The test frequencies included in the study were 10,25 , and $50 \mathrm{~Hz}$. This range of inputs was selected because they correspond to the frequency responsivity of cutaneous mechanoreceptors in the glabrous skin of the foot (15). All subjects were provided with a standard set of instructions and were asked to respond to the detection of a vibrotactile stimulus by depressing a handheld button immediately on perceiving the stimulus. A transistor logic signal generated by the handheld button was digitized concurrently with the vibratory stimulus.

An adapted staircase tracking method was used to establish an estimated detection threshold value for each test frequency and each stimulation site (2). This was accomplished by manually changing the signal amplitude in 1-dB increments based on the subject's perceptual response using a programmable logarithmic attenuator (Model PA 4; TuckerDavis, Alachua, FL). A positive detection trial was defined as a subject response, which fell between the midpoint of the vibratory burst and $2.5 \mathrm{~s}$ at the end of the sampling window. The lack of a subject response or a response, which fell outside the window defined for positive detection, was considered a negative detection trial. The investigator monitoring the digitized trial on a computer monitor was able to increase or decrease the test signal amplitude in real time based on positive or negative detection of the previous trial. In the case of this investigation, the $\mathrm{dB}$ level of the vibrotactile input served as the dependent variable, whereas group and site served as independent variables. Higher $\mathrm{dB}$ levels are indicative of higher detection thresholds and decreased or poor sensitivity. Conversely, lower $\mathrm{dB}$ levels are indicative of lower detection thresholds and increased or better sensitivity.

All stimuli were $1 \mathrm{~s}$ in total duration with 150 -ms linear rise-fall decay to eliminate mechanical transients during stimulus presentation. The intervals between stimuli varied randomly between 10 and $20 \mathrm{~s}$. The initial amplitude level for each test frequency at each test site was of supramaximal magnitude followed by signal attenuation in 6-dB descending steps until the first negative detection trial was recorded. The next stimulus was presented with a 6-dB increase until a positive detection was recorded. At this point, the test frequency signal amplitude was bracketed up or down using progressively smaller increments until a level was reached whereby half the stimuli were detected and oscillated around a change in amplitude of $1 \mathrm{~dB}$ for at least eight consecutive trials (Fig. 2). Detection threshold was achieved at each test frequency/stimulation site block within approximately 70 to 100 trials. The testing order of stimulation site and the order of test frequency were randomized across all subjects. The total time required to complete all assessment blocks was approximately $2.5 \mathrm{~h}$. Subjects were given a 10 - to $15-\mathrm{min}$ rest period after completing half of the assessment blocks to avoid concentration fatigue. To promote attentiveness, 


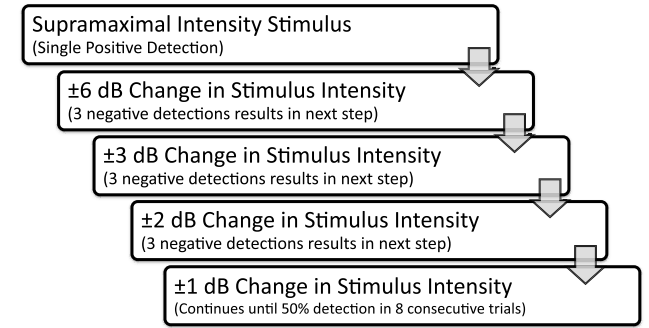

FIGURE 2-Flowchart of the adapted staircase method of manually adjusting vibrotactile input intensity to assess the vibrotactile detection threshold based on each subject's perceptual response.

subjects were verbally engaged by the investigators between testing blocks and encouraged to remain focused on the requirements of the task. To remove the possibility of any auditory cues associated with Minishaker apparatus, subjects wore headphones and were provided with pink noise throughout the protocol.

Data analysis. Probe displacement was measured offline from the digitized records to determine peak-to-peak displacement values in micrometers. A customized MatLab code (MathWorks, Inc., Natick, MA) identified the last eight trials of a given assessment block having four decision reversals to estimate vibrotactile detection threshold. Probe displacement measures from the positive detection trials with the last eight trials were averaged to obtain a single threshold estimate for each test frequency in $\mathrm{dB}$. Peak-topeak displacement measurements were performed on the middle $700 \mathrm{~ms}$ of the 1-s digitized LVDT waveform. Separate group (CAI, healthy)-by-site (head of first metatarsal, base of fifth metatarsal, heel) ANOVA were performed for the 10-, 25-, and 50- $\mathrm{Hz}$ test frequencies. In the presence of a significant group-by-site interaction or site main effect, post hoc Bonferroni tests were performed. Independent $t$-tests were conducted to determine whether group differences were present in age, height, or weight. The significance level for all analysis was set $a$ priori at $P \leq 0.05$. We opted not to perform any correction for multiple comparison on the $\alpha$ level to protect against making a type 1 error (26). Instead, effect sizes (ES) were calculated between groups using a bias-corrected Hedge's $g$ with corresponding 95\% confidence intervals (5).

\section{RESULTS}

No significant differences were identified in age, height, or weight between groups (all $P$ values $>0.07$ ). No significant group-by-site interactions were found at the $10-\mathrm{Hz}$ $(P=0.29$, observed power $(\mathrm{OP})=0.26)$, the $25-\mathrm{Hz}(P=0.70$, $\mathrm{OP}=0.10)$, or the $50-\mathrm{Hz}$ test frequencies $(P=0.98$, $\mathrm{OP}=0.05)$. However, a group main effect was present at $10 \mathrm{~Hz}(P<0.0001), 25 \mathrm{~Hz}(P=0.03)$, and $50 \mathrm{~Hz}(P=0.04)$, indicating that subjects with CAI had significantly higher detection thresholds when stimulation sites were pooled. In addition, a main effect for site was present at the $10-\mathrm{Hz}$ frequency $(P=0.01)$; however, main effects for site were not present at $25 \mathrm{~Hz}(P=0.17, \mathrm{OP}=0.36)$ or $50 \mathrm{~Hz}$ $(P=0.90, \mathrm{OP}=0.06)$. Post hoc comparisons at $10 \mathrm{~Hz}$ revealed that the heel had significantly higher detection thresholds when compared with the base of the fifth metatarsal $(P=0.008)$. However, no differences were present between the heel and the head of the first metatarsal $(P=0.98)$ or between the base of the fifth metatarsal and the head of the first metatarsal $(P=0.24)$. The means, SD, ES and ES confidence intervals for the detection thresholds of each group can be found in Table 1 .

\section{DISCUSSION}

The main finding of this study was that subjects with CAI exhibited higher vibrotactile detection thresholds, which indicated less sensitivity, compared with healthy subjects at the 10-, 25-, and 50-Hz frequencies. In the CAI group, lower plantar cutaneous sensitivity was not associated with any single stimulation site. Rather, all three stimulation sites demonstrated higher detection thresholds for each respective frequency in subjects with CAI compared with healthy individuals. The most robust difference between groups was found at $10 \mathrm{~Hz}$ as indicated by large ES ranging from 1.89 to 2.36 , with corresponding $95 \%$ confidence intervals that do not encompass zero. Differences in sensitivity of the plantar cutaneous receptors may help explain several of the functional impairments previously associated with CAI.

The stimulation sites tested in this study receive sensory innervations from the sural nerve and branches of the tibial nerve including the medial plantar, lateral plantar, and

TABLE 1. Means \pm SD (dB), ES, and ES 95\% confidence intervals (CI) for vibrotactile detection thresholds for the CAI and healthy groups at the 10-, 25-, and 50-Hz test frequencies.

\begin{tabular}{|c|c|c|c|c|c|}
\hline Frequency & Site & CAI Group & Healthy Group & ES & $95 \% \mathrm{Cl}$ \\
\hline \multirow[t]{3}{*}{$10 \mathrm{~Hz}^{b}$} & $\mathrm{Heel}^{c}$ & $47.23 \pm 3.61^{a}$ & $39.35 \pm 4.05$ & 1.89 & 0.62 to 3.16 \\
\hline & Fifth metatarsal & $43.67 \pm 4.49^{a}$ & $35.40 \pm 3.17$ & 2.04 & 0.74 to 3.34 \\
\hline & First metatarsal & $47.71 \pm 4.68^{a}$ & $36.31 \pm 4.37$ & 2.36 & 0.98 to 3.73 \\
\hline \multirow[t]{3}{*}{$25 \mathrm{~Hz}^{b}$} & Heel & $36.89 \pm 3.25$ & $32.93 \pm 4.60$ & 0.94 & -0.09 to 1.97 \\
\hline & Fifth metatarsal & $37.29 \pm 5.82$ & $31.82 \pm 5.56$ & 0.91 & -0.12 to 1.94 \\
\hline & First metatarsal & $35.57 \pm 4.55$ & $29.31 \pm 7.51$ & 0.95 & -0.08 to 1.99 \\
\hline \multirow[t]{3}{*}{$50 \mathrm{~Hz}^{b}$} & Heel & $27.26 \pm 4.43$ & $21.51 \pm 5.10$ & 1.14 & 0.08 to 2.20 \\
\hline & Fifth metatarsal & $27.76 \pm 5.84$ & $21.99 \pm 7.52$ & 0.81 & -0.21 to 1.83 \\
\hline & First metatarsal & $26.13 \pm 8.45$ & $21.18 \pm 7.12$ & 0.60 & -0.40 to 1.60 \\
\hline
\end{tabular}

Higher $\mathrm{dB}$ levels represent greater stimulus amplitudes and higher vibrotactile detection thresholds or decreased sensitivity.

${ }^{a}$ Represents values for a sample size of $n=6$.

${ }^{b}$ Represents a group main effect in which subjects with CAI had higher thresholds than healthy control subjects.

${ }^{c}$ Represents a site main effect in which the heel had higher thresholds than the base of the fifth metatarsal. 
calcaneal nerves (34). Although sural and tibial nerve injuries can occur in conjunction with ankle sprains (14), it is unlikely that direct nerve trauma is the source of the somatosensory alterations in this investigation because deficits were only demonstrated at the lower test frequencies. Deficits at the $10-$ to $50-\mathrm{Hz}$ frequencies would indicate that the involved mechanoreceptors include Ruffini organs, Merkel disks, and Meissner corpuscles (37). Pacinian corpuscles are stimulated at much higher frequencies $(200 \mathrm{~Hz})$ and seemed to behave similarly in both groups based on the thresholds attained from higher test frequencies $(125$ and $250 \mathrm{~Hz})$, which were explored in the early stages of data collection and later abandoned. This is further supported by a linear trend in $P$ values and ES exhibited across the $10-\mathrm{Hz}$ $(P<0.0001$, average ES $=2.10), 25-\mathrm{Hz}(P=0.03$, average $\mathrm{ES}=0.93)$, and $50-\mathrm{Hz}(P=0.04$, average $\mathrm{ES}=0.85)$ test frequencies. This suggests that the lower plantar cutaneous sensitivity exhibited by the CAI group is contextually dependent on the frequency of the stimulus rather than location. These changes in sensation may be associated with alterations in spinal or cortical levels of the central nervous system after joint injury, which may influence the behavior of peripheral afferent receptors. This is supported by recent studies $(31,32)$, which identified deficits in cutaneous vibratory detection threshold in individuals with hip and knee osteoarthritis at multiple sites on the lower extremity as well as the radial tuberosity on the upper extremity. This indicates that lower extremity pathologic abnormalities may be associated with global changes in cutaneous sensation in response to joint trauma. Because this study is limited to detection thresholds on the plantar surface, future research should examine if the higher detection thresholds associated with CAI are local or global in nature.

Examining the extent to which sensory alterations occur may also elucidate the underlying mechanism of these sensory deficits. Unlike the causes for desensitization of plantar cutaneous mechanoreceptors associated with neurodegenerative health conditions or normal aging (30), the underlying mechanism for decreased plantar cutaneous vibratory acuity in people with CAI is unclear. However, it can be speculated that these sensory alterations may be the result of presynaptic inhibition of cutaneous mechanoreceptors from descending pathways of the central nervous system to reduce the large amount of sensory information coming from these receptors (33). This would allow the sensorimotor system to focus on critical events requiring immediate attention to maintain gait and posture; however, it may have deleterious implications on the overall quality of the motor output, which may contribute to ongoing recurrent sprains and/or episodes of giving way that people with CAI often experience. It may be that the behavior observed in this study is capturing a unique impairment or sensorimotor adaptation associated with CAI. Although no direct connection can be made between sensorimotor function and plantar cutaneous detection thresholds in this investigation, those with CAI reported reduced functional capacity during the previous week on the FAAM and the FAAM Sport self-reported function scales.

Lower vibrotactile sensitivity at frequencies ranging from 10 to $50 \mathrm{~Hz}$ may provide insight into functional impairments in gait and postural control because these inputs closely correspond with the spectral bandwidth of frequencies associated with these tasks (10). Recent meta-analyses have concluded that both dynamic and static postural control is decreased in individuals with CAI $(3,39)$. In addition, studies investigating gait initiation and termination have identified alterations in feed-forward and feedback neuromuscular control $(11,38)$. Although several impairments may contribute to these previous findings, the results of this current investigation suggest that these deficiencies could be linked to altered plantar cutaneous somatosensation. Future research should systematically evaluate the relationship between altered plantar cutaneous sensitivity and sensorimotor system impairments in people with CAI.

Changes in cutaneous afferent input may also have a role in regulating $\alpha$-motoneuron pool excitability in the lower extremity (29). There is a clear relationship between plantar cutaneous mechanoreceptors and soleus $\alpha$-motoneuron pool excitability (29). Increased stimulation to the plantar receptors of the heel produced a significant facilitation in the soleus motoneuron pool excitability of healthy adults (29). It has been established that people with CAI exhibit soleus $\alpha$-motoneuron pool inhibition (20). Soleus muscle inhibition associated with CAI had been purported to be related to ankle joint trauma (20); however, the inhibition may be arising from a loss of essential noise from the plantar cutaneous receptors. In this study, we found that subjects with CAI had significantly reduced detection sensitivity, specifically around the heel. Alterations in $\alpha$-motoneuron pool excitability and concurrent changes in cutaneous sensation have not been investigated after ankle injury. Coexisting changes in neuromuscular function and sensory input could contribute to a decreased ability to create both local and global defense mechanisms in the presence of unexpected inversion or supination (9). Future exploration in this area is warranted because establishing the relationship between sensory alterations and sensorimotor function may facilitate the development of new interventions to address the functional impairments exhibited by CAI.

This study was not without limitations. Based on the retrospective design, we are unable to establish a causal relationship between CAI and decreased plantar cutaneous sensitivity. Future studies should prospectively investigate changes in plantar cutaneous sensation after ankle sprains. Because detection threshold was the only dependent measure in this investigation, we are unable to directly link the findings of this investigation with previously identified decreases in postural control or alterations in gait. In addition, detection threshold assessment is based on perceptual responses and although achieving the detection threshold is based on multiple responses using the adapted staircase method of introducing vibrotactile inputs, it is unknown if 
any additional confounders were present, which may affect the results. Also, this study did not measure vibrotactile acuity in the uninvolved limb. A bilateral, within-subject comparison was not collected in this study because of its exploratory nature and because several subjects demonstrated bilateral CAI. As previously mentioned, future studies should determine whether these changes are localized to the involved limb or if alterations in cutaneous sensation occur globally.

Finally, the sample included in this study was relatively small. Although an a priori power analysis was not conducted, post hoc power analyses determined we had ample observed power to detect group effects (OPs $=0.57-0.99)$; however, for our nonsignificant interactions, the observed power was very low $(\mathrm{OPs}=0.05-0.26)$. This indicates that if the ES were to remain constant, the number of subjects required to detect group differences at individual stimulation sites would require a very large sample size $(<200$ per group), which would be unrealistic for psychometric testing if these differences were to exist. We believe that the addition of the ES and 95\% CI strengthen our findings by highlighting the magnitude of difference between groups and also the similarity in behavior exhibited across stimulation sites in each group for each respective frequency. In

\section{REFERENCES}

1. Anandacoomarasamy A, Barnsley L. Long term outcomes of inversion ankle injuries. Br J Sport Med. 2005;39(3):e14.

2. Andreatta RD, Davidow JH. Mechanical frequency and stimulation-site-related differences in vibrotactile detection capacity along the lip vermilion in young adults. Clin Oral Investig. 2006; 10(1):17-22.

3. Arnold BL, De La Motte S, Linens S, Ross SE. Ankle instability is associated with balance impairments: a meta-analysis. Med Sci Sports Exerc. 2009;41(5):1048-62.

4. Bolanowski SJ, Gescheider GA, Verillo RT, Checkosky CM. Four channels mediate the mechanical aspects of touch. $J$ Acoust Soc Am. 1988;84(5):1680-94.

5. Borenstein M, Hedges LV, Higgins JPT, Rothstein HR. Introduction to Meta-analysis. West Sussex (UK): John Wiley \& Sons, Ltd.; 2009. p. 21-32.

6. Bullock-Saxton JE. Local sensation changes and altered hip muscle function following severe ankle sprain. Phys Ther. 1994;74(1): 17-31.

7. Davids K, Shuttleworth R, Button C, Renshaw I, Glazier P. "Essential noise" - enhancing variability of informational constraints benefits movement control: a comment on Waddington and Adams (2003). Br J Sports Med. 2004;38(5):601-5.

8. Docherty CL, Gansneder BM, Arnold BL, Hurwitz SR. Development and reliability of the ankle instability instrument. $J$ Athl Train. 2006;41(2):154-8.

9. Duysens J, Levin O. Ankle sprains: getting off on the wrong foot. Exer Sport Sci Rev. 2010;38(3):143-9.

10. Harris GF, Acharya KR, Bachschmidt RA. Investigation of spectral content from discrete plantar areas during adult gait: an expansion of rehabilitation technology. IEEE Trans Rehabil Eng. 1996;4(4):360-74.

11. Hass CJ, Bishop MD, Doidge D, Wikstrom EA. Chronic ankle instability alters central organization of movement. Am J Sports Med. 2010;38(4):829-34. some cases, the $95 \%$ CI narrowly encompassed zero, which may be remedied by increasing sample size. However, when cumulatively examining the ES in this study, there was an obvious trend toward the strong group effects.

In conclusion, the results of this study indicate that individuals with CAI demonstrate higher plantar cutaneous vibrotactile detection thresholds or decreased sensitivity on the plantar surface when compared with adults with no history of ankle sprain. These findings were associated with test frequencies ranging from 10 to $50 \mathrm{~Hz}$, which may shed insight into the common functional impairments previously identified in those with CAI. Future research is needed to determine the role of altered plantar cutaneous somatosensation in the development and rehabilitation of CAI.

This study was funded by the University of Kentucky College of Health Sciences Office of Research. All data were collected at the University of Kentucky.

No conflicts of interest were associated with the authors and the results of this research.

The results of the present study do not constitute endorsement by the American College of Sports Medicine.

12. Hertel J. Functional anatomy, pathomechanics, and pathophysiology of lateral ankle instability. J Athl Train. 2002;37(4):364-75.

13. Hootman JM, Dick R, Agel J. Epidemiology of collegiate injuries for 15 sports: summary and recommendations for injury prevention initiatives. J Athl Train. 2007;42(2):311-9.

14. Hunt GC. Injuries of peripheral nerves of the leg, foot and ankle: an often unrecognized consequence of ankle sprains. Foot. 2003; 13(1):14-8

15. Kennedy PM, Inglis JT. Distribution and behaviour of glabrous cutaneous receptors in the human foot sole. J Physiol. 2002;538 (Pt 3):995-1002.

16. Liu W, Lipsitz LA, Montero-Odasso M, Bean J, Kerrigan DC, Collins JJ. Noise-enhanced vibrotactile sensitivity in older adults, patients with stroke, and patients with diabetic neuropathy. Arch Phys Med Rehabil. 2002;83(2):171-6.

17. Martin RL, Irrgang JJ, Burdett RG, Conti SF, Van Swearingen JM. Evidence of validity for the Foot and Ankle Ability Measure (FAAM). Foot Ankle Int. 2005;26(11):968-83.

18. McKeon PO, Booi MJ, Branam B, Johnson DL, Mattacola CG. Lateral ankle ligament anesthesia signficantly alters single limb postural control. Gait Posture. 2010;32(3):374-7.

19. McKeon PO, Hertel J. Spatiotemporal postural control deficits are present in those with chronic ankle instability. BMC Musculoskelet Disord. 2008;9:76.

20. McVey ED, Palmieri RM, Docherty CL, Zinder SM, Ingersoll CD. Arthrogenic muscle inhibition in the leg muscles of subjects exhibiting functional ankle instability. Foot Ankle Int. 2005;26(12): 1055-61.

21. Meyer PF, Oddsson LIE, De Luca CJ. The role of plantar cutaneous sensation in unperturbed stance. Exp Brain Res. 2004;156(4): 505-12.

22. Myers JB, Riemann BL, Hwang J, Fu FH, Lephart SM. Effect of peripheral afferent alteration of the lateral ankle ligaments on dynamic stability. Amer J Sports Med. 2003;31(4):498-506. 
23. Nashner LM. Adapting reflexes controlling the human posture. Exp Brain Res. 1976;26(1):59-72.

24. Nigg BM, Nurse MA, Stefanyshyn DJ. Shoe inserts and orthotics for sport and physical activities. Med Sci Sports Exerc. 1999; 31(7 suppl):S421-8.

25. Nyska M, Shabat S, Simkin A, Neeb M, Matan Y, Mann G. Dynamic force distribution during level walking under the feet of patients with chronic ankle instability. Br J Sports Med. 2003;37(6):495-7.

26. Perneger TV. What's wrong with Bonferroni adjustments. $\mathrm{Br}$ Med J. 1998;316(7139):1236-8.

27. Perry SD. Evaluation of age-related plantar-surface insensitivity and onset age of advanced insensitivity in older adults using vibratory and touch sensation tests. Neurosci Lett. 2006;392(1-2):62-7.

28. Pope M, Chinn L, Mullineaux D, McKeon PO, Drewes L, Hertel J. Spatial postural control alterations with chronic ankle instability. Gait Posture. 2011;34(2):154-8.

29. Sayenko DG, Vette AH, Obata H, Alekhina MI, Akai M, Nakazawa K. Differential effects of plantar cutaneous afferent excitation on soleus stretch and H-reflex. Muscle Nerve. 2009; 39(6):761-9.

30. Shaffer SW, Harrison AL. Aging of the somatosensory system: a translational perspective. Phys Ther. 2007;87(2):193-207.

31. Shakoor N, Agrawal A, Block JA. Reduced lower extremity vibratory perception in osteoarthritis of the knee. Arthritis Rheum. 2008;59(1):117-21.

32. Shakoor N, Lee KJ, Fogg LF, Block JA. Generalized vibratory deficits in osteoarthritis of the hip. Arthritis Rheum. 2008;59(9): $1237-40$
33. Squire LR, Berg D, Bloom FE, du Lac S, Ghosh A, Spitzer NC. Fundamental Neuroscience. 3rd ed. Burlington (MA): Elsevier; 2008. p. 581-608.

34. Unlü RE, Orbay H, Kerem M, Esmer AF, Tüccar E, Sensöz O. Innervation of three weight-bearing areas of the foot: an anatomic study and clinical implications. J Plast Reconstr Aesthet Surg. 2008;61(5):557-61.

35. Valderrabano V, Hintermann B, Horisberger M, Fung TS. Ligamentous posttraumatic ankle osteoarthritis. Am J Sports Med. 2006; 34(4):612-20.

36. van Deursen RW, Sanchez MM, Derr JA, Becker MB, Ulbrecht JS, Cavanagh PR. Vibration perception threshold testing in patients with diabetic neuropathy: ceiling effects and reliability. Diabet Med. 2001;18(6):469-75.

37. Wells C, Ward LM, Chua R, Inglis JT. Regional variation and changes with ageing in vibrotactile sensitivity in the human footsole. J Gerontol A Biol Sci Med Sci. 2003;58(8):680-6.

38. Wikstrom E, Bishop M, Inamdar A, Hass C. Gait termination control strategies are altered in chronic ankle instability subjects. Med Sci Sports Exerc. 2010;42(1):197-205.

39. Wikstrom EA, Naik S, Lodha N, Cauraugh JH. Balance capabilities after lateral ankle trauma and intervention: a meta-analysis. Med Sci Sports Exerc. 2009;41(6):1287-95.

40. Wikstrom EA, Tillman MD, Chmielewski TL, Cauraugh JH, Naugle KE, Borsa PA. Self-assessed disability and functional performance in individuals with and without ankle instability: a case control study. J Orthop Sport Phys Ther. 2009;39(6):458-67. 